\title{
Science journals and global outreach initiatives in developing countries
}

Donald R. Miller MD FRCPC

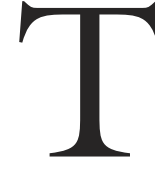

HE Journal is proud to be member of the Council of Science Editors (CSE), which is an international organization devoted to promotion of excellence in the communication of scientific information through development of editorial policies, scientific and educational meetings, and specialized publications. The Council ${ }^{\mathrm{A}}$ serves an invaluable role in providing authoritative resources on current and emerging issues in the communication of scientific information.

In January 2005, the CSE established a task force to encourage scientific journals to assume a role in combating poverty and disease, and to work towards sustainable development. Subsequently in 2006, the CSE called for a global theme issue amongst journals to address the subject of poverty and human development. Since that time, the editors representing 224 scientific publications throughout the world, including the Journal, have agreed to participate by simultaneously publishing papers on this topic of worldwide interest in order to raise awareness, stimulate interest, and stimulate research into poverty and human development. This initiative represents an international collaboration amongst journals from both developed and developing countries. Recently, excitement for this initiative was further enhanced through collaboration of the CSE and the Fogarty International Center and the National Library of Medicine of the National Institutes of Health to co-sponsor a presentation from select groups of authors/researchers undertaking original research related to the Global Theme Issues.

Participating journals have agreed to publish a variety of materials, including original research papers, review articles, editorials, perspectives, news stories, and other types of articles on the subject of poverty

A Council of Science Editors; http://www.councilscienceeditors. org/ (accessed August 22, 2007). and human development with a common publication or release date of October 22, 2007. While some journals have dedicated an entire issue to this subject, others have published selected papers. In this issue of the Journal, we have invited Dr. Enright, Chair of the Canadian Anesthesiologists' Society International Education Fund (CASIEF) to share with us the initial experience from an international overseas anesthesia training program in one of the world's poorest countries - Rwanda.

As reported by Dr. Enright, ${ }^{1}$ a program leading to a Master's degree in anesthesia was established in 2006 in Rwanda as a joint venture between the National University of Rwanda, the CASIEF, and the American Society of Anesthesiologists' Overseas Teaching Programme (ASAOTP). It is rewarding to observe the partnering of these organizations to coordinate their resources on a new overseas medical training initiative. It may not be surprising to learn of the challenges which now exist in recruiting residents, and in developing an academic culture and evaluation system. Inadequate equipment and drug shortages limit the types of anesthesia provided, and limited biomedical support provides additional ongoing challenges.

Through the program in Rwanda, volunteer Canadian and American anesthesiologists now provide regular instruction in the operating room and also in the classroom setting. While the focus is on residents in anesthesia, the volunteers also teach the nurse anesthetists. Since the time of program implementation considerable progress has been made and there are further initiatives underway to improving the management of acute and chronic pain. In time, it is hoped that Rwanda will become self-sufficient in training its own anesthesiologists and in retaining them to provide anesthesia services throughout the country, using a template which has been highly successful with the CASIEF initiative in Nepal. ${ }^{2-4}$

CAN J ANESTH $2007 / 54: 11 /$ pp 868-871

From the Department of Anesthesia, University of Ottawa, Ottawa, Ontario, Canada. Address correspondence to: Dr. Donald Miller, Department of Anesthesia, The Ottawa Hospital, General Campus, Room 1409, 501 Smyth Road, Ottawa, Ontario K1H 8L6, Canada. Phone: 450-477-7607; Fax: 450-477-8472; E-mail: cja_office@cas.ca 
In contrast to a program development approach, a second special article in this issue focuses on the challenges, logistics and benefits of providing temporary anesthesia services in areas of developing countries which have extremely limited access to anesthetic and surgical services. In this article, ${ }^{5}$ Dr. Froese reflects from her personal experiences in developing countries in identifying the ongoing need for anesthesiologists to be involved in short-term surgical projects in areas of greatest need, as a result of woefully inadequate number of physicians and their geographic maldistribution. While the lack of safe anesthesia services is known to severely limit the performance of needed surgical procedures in developing countries, even where well-trained anesthesiologists are available in major urban centres, resources are often absent or limited for large numbers of people in rural or remote areas. Special service anesthesia projects have taken place in Central and South America, Africa, Asia and Eastern Europe.

The severe shortages of trained health professionals which plague developing countries often reflect complex economic and political problems that will require international cooperation and considerable resources for their ultimate resolution. Dr. Froese points out rather convincingly ${ }^{5}$ that until such time as surgical services are widely available and affordable in remote as well as urban areas of developing countries, anesthesiologists can continue to provide a valuable and personally-rewarding contribution through short-term assistance. Opportunities are available for members of our specialty to enhance our unique contributions to developing countries in this manner.

Scientific journals can outreach in ways other than through special initiatives such as the global theme issue on poverty and human development. In the spring of 2007, the Journal became a partner of the Health InterNetwork Access to Research Initiative $^{\mathrm{B}}$ (HINARI) which was established by the World Health Organization in partnership with major publishers, in order that developing countries could gain free access to a large collection of the world's health and biomedical literature. Currently, more than 3,700 journals are listed through HINARI in 113 countries, providing unlimited free access of their scientific content to health workers and researchers. The overall aim of the initiative is to improve world health by eliminating subscription barriers in accessing the contemporary body of peer reviewed scientific

B Health InterNetwork Access to Research Initiative: http:// www.who.int/hinari/en/ (accessed August 22, 2007). material which is instrumental in providing the latest information on disease mechanisms, diagnosis and treatment. It is hoped that with increasing internet access in developing countries (still a major problem in many jurisdictions) that the HINARI initiative will have an increasingly profound effect in all developing countries.

Finally, the commitment of the Journal towards global outreach initiatives extends beyond the publication of the special articles in this issue. We shall welcome, in consideration for publication, letters to the editor commenting on these issues, as well as relevant original articles addressing the subject of poverty and human development. Anesthesiologists and our specialty journals can make a unique contribution to health-related issues in the developing world. The need to address these world health challenges has never been greater.

\section{Les revues scientifiques et les initiatives mondia- les d'aide aux pays en voie de développement}

Le Journal est fier d'être membre du Council of Science Editors (CSE), une organisation internationale qui se consacre à la promotion de l'excellence dans la communication de renseignements scientifiques par le développement de politiques rédactionnelles, de conférences scientifiques et éducationnelles, et par des publications spécialisées. Le Councił ${ }^{\AA}$ joue un rôle prépondérant en fournissant des ressources dignes de foi concernant les questions actuelles et émergentes qui touchent à la communication de renseignements scientifiques.

En janvier 2005, le CSE a créé un groupe de travail afin d'encourager les revues scientifiques à jouer un rôle dans le combat contre la pauvreté et la maladie, et de travailler dans le sens du développement durable. Par la suite, en 2006, le CSE a proposé aux revues de consacrer un numéro à un thème international afin de se pencher sur la question de la pauvreté et du développement humain. Depuis, les rédacteurs, représentant 224 publications scientifiques du monde entier, y compris le Journal, ont consenti à participer à cette initiative en publiant simultanément 
des articles sur ce sujet d'intérêt mondial afin de promouvoir la conscientisation, d'aiguiser l'intérêt et de stimuler la recherche sur la pauvreté et le développement humain. Cette initiative est le fruit d'une collaboration internationale des revues des pays développés et en voie de développement. L'excitation liée à cette initiative a récemment connu un bond supplémentaire en raison de la collaboration du CSE et du Fogarty International Center ainsi que de la National Library of Medicine des Instituts nationaux de santé. En effet, ces institutions vont co-financer une présentation de groupes choisis d'auteurs/de chercheurs qui entreprennent des recherches originales en relation avec les Questions sur un sujet d'intérêt international.

Les revues participantes ont consenti à publier différents types d'articles, dont des comptes-rendus de recherche originaux, des articles de synthèse, des éditoriaux, des perspectives, des nouvelles ainsi que d'autres types d'articles traitant de la pauvreté et du développement humain et ce, à une date de publication commune, le 22 octobre 2007. Alors que certaines revues ont consacré un numéro entier au sujet, d'autres ont publié des articles sélectionnés. Dans ce numéro du Journal, nous avons invité la Dre Enright, présidente du Fonds d'éducation internationale de la Société canadienne des anesthésiologistes (FÉI SCA), à partager avec les lecteurs l'expérience initiale d'un programme de formation en anesthésie international à l'étranger, dans l'un des pays les plus pauvres, le Rwanda.

Comme le rapporte la Dre Enright, un programme menant à une maîtrise en anesthésie a été mis en place en 2006 au Rwanda en tant que projet conjoint de la National University of Rwanda, la FÉI SCA et le American Society of Anesthesiologists' Overseas Teaching Programme (ASAOTP - Programme d'enseignement à l'étranger de la Société américaine des anesthésiologistes). Il est gratifiant d'observer la collaboration de ces organisations afin de coordonner leurs ressources pour une nouvelle initiative de formation médicale à l'étranger. Sans grande surprise, l'article de la Dre Enright nous fait part des défis qui existent actuellement dans le recrutement de résidents ainsi que dans le développement d'une culture universitaire et d'un système d'évaluation. Le matériel inadapté et les pénuries de médicaments limitent les types d'anesthésie fournis aux patients; en outre, un soutien biomédical limité crée continuellement des défis supplémentaires.

Par le biais du programme au Rwanda, des anesthésiologistes canadiens et américains bénévoles fournissent désormais un enseignement régulier en salle d'opération, mais également dans un cadre de salle de classe. Bien que l'emphase soit mise sur les résidents en anesthésie, les bénévoles enseignent également aux infirmiers et infirmières en anesthésie. Depuis la mise en ouvre du programme, des progrès considérables ont été faits; de plus, d'autres initiatives sont en cours afin d'améliorer la prise en charge de la douleur aiguë et chronique. Nous espérons qu'avec le temps le Rwanda deviendra autosuffisant dans la formation de ses propres anesthésiologistes et pourra les garder afin de fournir des services d'anesthésie partout dans le pays, sur la base d'un programme modèle très réussi de la FÉI SCA au Népal. ${ }^{2-4}$

En contraste à l'approche du programme de développement, le deuxième article spécial de ce numéro se concentre sur les défis, la logistique nécessaire et les avantages de l'offre de services d'anesthésie temporaires dans les zones des pays en voie de développement qui n'ont qu'un accès extrêmement limité à des services anesthésiques et chirurgicaux. Dans cet article, ${ }^{5}$ la Dre Froese tire des conclusions de son expérience personnelle dans les pays en voie de développement concernant l'identification $\mathrm{du}$ besoin continu d'anesthésiologistes s'engageant dans des projets chirurgicaux à court terme dans les zones aux besoins les plus urgents, en raison du nombre extrêmement inadéquat de médecins et de leur mauvaise distribution géographique. Alors que nous savons que le manque de services anesthésiques sécuritaires limite de façon draconienne la réalisation d'interventions chirurgicales nécessaires dans les pays en voie de développement, même dans les centres urbains les plus importants, où des anesthésiologistes bien formés existent, les ressources font souvent défaut ou sont limitées pour une population importante dans les zones rurales ou reculées. Des projets spéciaux de services d'anesthésie ont eu lieu en Amérique du Sud, en Amérique Centrale, en Afrique, en Asie et en Europe de l'Est.

Les pénuries importantes en professionnels de la santé formés qui frappent les pays en voie de développement sont souvent le reflet de problèmes économiques et politiques complexes. Ceux-ci nécessiteront une coopération internationale et des ressources considérables pour être finalement réglés. La Dre Froese souligne de façon très convaincante ${ }^{5}$ qu'en attendant que les services chirurgicaux soient facilement disponibles et abordables dans les zones reculées aussi bien qu'urbaines des pays en voie de développement, les anesthésiologistes peuvent continuer à fournir une contribution précieuse et gratifiante par le biais d'une assistance à court terme. Il existe des opportunités pour les membres de 
notre spécialité afin d'améliorer de cette façon notre contribution exceptionnelle aux pays en voie de développement.

Il existe d'autres façons pour les revues scientifiques d'intervenir que par le biais d'initiatives spéciales telles que la question portant sur le thème international de la pauvreté et du développement humain. Au printemps 2007, le Journal s'est associé comme partenaire au Health InterNetwork Access to Research Initiative $^{B}$ (HINARI), qui a été fondé par l'Organisation mondiale de la Santé en collaboration avec certaines des maisons d'édition les plus importantes, afin que les pays en voie de développement bénéficient d'un accès gratuit à une vaste collection de la littérature mondiale en santé et en recherche biomédicale. Actuellement, plus de 3700 revues sont inscrites via HINARI dans 113 pays, fournissant aux travailleurs et aux chercheurs dans le domaine de la santé un accès gratuit illimité à leur contenu scientifique. L'objectif global de cette initiative est l'amélioration de la santé au niveau mondial par l'élimination des barrières que peuvent constituer les abonnements pour l'accès au corpus contemporain de matériel scientifique revu par des pairs, lequel joue un rôle crucial en fournissant les renseignements les plus récents sur les mécanismes des maladies, les diagnostics et les traitements. Nous espérons que, grâce à l'accès grandissant à Internet dans les pays en voie de développement - ce qui demeure un problème majeur dans de nombreuses juridictions - l'initiative HINARI aura un effet de plus en plus profond dans tous les pays en voie de développement.

En conclusion, l'engagement du Journal envers les initiatives mondiales d'aide va au-delà de la publication d'articles spéciaux dans ce numéro. Nous serons heureux de recevoir, avec intention de publier, des lettres à la rédaction commentant ces questions ainsi que des articles originaux pertinents s'adressant aux questions de la pauvreté et du développement humain. Les anesthésiologistes et nos revues spécialisées peuvent contribuer de manière exceptionnelle aux questions liées à la santé dans les pays en voie de développement. Le besoin de relever ces défis de la santé au niveau mondial n'a jamais été aussi important.

\section{References}

1 Enright A. Anesthesia training in Rwanda. Can J Anesth 2007; 54: 935-9.

2 Tweed WA, Amatya R, Tuladhar TM, Maltby JR, Gurung CK, McCaughey TJ. Anaesthesia services and the education of anaesthetists in Nepal: a model for sustainable development? Can J Anaesth 1993; 40: 993-9.

3 Maltby JR, Amatya R, Rana NB, Shrestha BM,
Tuladbar TM, McCaughey TJ. Anaesthesia training and development in Nepal 1985-1990. Can J Anaesth 1991; 38: 105-10.

4 Shrestha BM, Rana NB. Training and development of anesthesia in Nepal - 1985 to 2005. Can J Anesth 2006; 53: 339-43.

5 Froese $A$. Anesthesia and the role of short-term service delivery in developing countries. Can J Anesth 2007; 54: 940-6. 